\title{
ELECTRIC FIELD EFFECTS ON NEMATIC WETTING LAYERS
}

\author{
G. KELLY, N. J. MOTTRAM, A. RAMAGE \\ Department of Mathematics, University of Strathclyde, \\ Livingstone Tower, Glasgow G1 1XH, U.K.
}

We present a theoretical investigation of the temperature and electric field dependence of nematic liquid crystal wetting layers close to an aligning substrate within a confined system. Using a mesoscopic $\mathbf{Q}$ tensor theory coupled to Maxwell's equations for the electric field, we consider the existence and stability of homogeneous nematic wetting layers close to the substrate. Numerical results are presented showing the phase diagram for the isotropic (paranematic), nematic and wetting layer states. The effect on the isotropicwetting transition, from first order to second order, when an electric field is applied is then investigated.

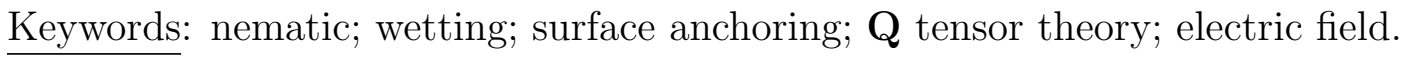

\section{INTRODUCTION}

Liquid crystal devices usually require that bounding substrates induce uniform alignment, and for this reason there has been intense interest in the technology of nematic alignment at solid surfaces. The reduction in symmetry, induced order and alignment of the director close to a substrate greatly affects the behaviour of a nematic and may compete with the bulk liquid crystal configuration. An enhancement of the order parameter near the substrate may induce a paranematic state even at temperatures well above the nematic to isotropic transition point. Such surface ordering may also be sufficient to cause the growth of a wetting layer of nematic between the isotropic phase in the bulk of the cell and the substrate [1]. The growth of the wetting layer near to $T_{N I}$, the clearing point temperature, where the isotropic and nematic states are of equal energy, is controlled by the competition between the surface anchoring and the energy of the nematic-isotropic interface.

Previous work has shown that, in a semi-infinite nematic sample bounded by an aligning substrate, the first order wetting layer phase transition occurs at higher temperatures as an applied field increases [2]. For sufficiently large applied fields the transition from the paranematic state to complete wetting becomes continuous. However, work in this area has assumed that the applied field is constant and not affected by the nematic orientation or order parameter [1-3]. This is either because a magnetic field was applied which remains constant due to the low magnetic susceptiblity, or because an electric field was applied perpendicular to the direction of director and order variations. 
In this paper, we consider a sample of liquid crystal sandwiched between two substrates which are fixed in the $x y$-plane (see Figure 1). It is assumed that the two substrates are treated so that they induce director alignment in the $x$-direction and will tend to induce order in an otherwise isotropic system. Although in the theoretical model the director is not constrained, we find that it remains in the $x$ direction throughout the cell. We consider a material with a negative dielectric permittivity in the nematic phase so that, when an electric field is applied across the cell in the $z$ direction, the field induces order in directions perpendicular to $z$. We therefore expect that a reduction in temperature or an increase in field strength will induce order and the system will undergo a transition to the nematic phase. Investigating the nature of this transition and the possibility of an intermediate (wetting) state will be the main aim of this paper.

For such a system, where spatial changes in order are important, it is necessary to use the Landau-de Gennes $\mathbf{Q}$ tensor theory [4]. The $\mathbf{Q}$ tensor is derived from the second moment of the molecular orientational distribution function and contains information about the directors (the eigenvectors of $\mathbf{Q}$ ) and the order in the direction of the directors (the corresponding eigenvalues of $\mathbf{Q}$ ). This theory is outlined below.

\section{LANDAU-DE GENNES THEORY}

The symmetry of the orientational probability distribution, $f$, of nematic liquid crystal molecules is such that $f(\mathbf{l})=f(-\mathbf{l})$, where $\mathbf{l}$ is the vector along the molecular long axis. Therefore, the first moment of this distribution is zero and a useful measure of order in such a system is the second moment, which is proportional to $\mathbf{l} \otimes \mathbf{l}$ [4]. Subtracting the appropriate multiple of the identity matrix forms the symmetric traceless tensor order parameter $\mathbf{Q}$,

$$
\mathbf{Q}=\left(\begin{array}{ccc}
q_{1} & q_{2} & q_{3} \\
q_{2} & q_{4} & q_{5} \\
q_{3} & q_{5} & -q_{1}-q_{4}
\end{array}\right)
$$

In more physically understandable terms, we can express $\mathbf{Q}$ in terms of two directors, the unit vectors $\mathbf{n}$ and $\mathbf{m}$, and two order parameters, $S_{1}$ and $S_{2}$, as

$$
\mathbf{Q}=S_{1}(\mathbf{n} \otimes \mathbf{n})+S_{2}(\mathbf{m} \otimes \mathbf{m})-\frac{1}{3}\left(S_{1}+S_{2}\right) \mathbf{I}
$$

where $\mathbf{I}$ is the identity matrix. The vectors $\mathbf{n}$ and $\mathbf{m}$ are then two of the eigenvectors of $\mathbf{Q}$, the third being $\mathbf{n} \times \mathbf{m}$, and the corresponding eigenvalues are $\left(2 S_{1}-S_{2}\right) / 3,\left(2 S_{2}-S_{1}\right) / 3$ and $-\left(S_{1}+S_{2}\right) / 3$, respectively. Note that in a uniaxial system two of the eigenvalues are equal so either $S_{1}=0, S_{2}=0$ or $S_{1}=S_{2}$ and the largest eigenvalue is $2 S / 3$, where $S$ is either $S_{1}$ or $S_{2}$.

In our system we will see that the director $\mathbf{n}$ remains in the $x$ direction (and secondary 
director $\mathbf{m}$ remains in the $y$ direction). In this case, $\mathbf{Q}$ is always of the form

$$
\mathbf{Q}=\left(\begin{array}{ccc}
q_{1} & 0 & 0 \\
0 & q_{4} & 0 \\
0 & 0 & -q_{1}-q_{4}
\end{array}\right)
$$

and the eigenvalue associated with $\mathbf{n}$ is $q_{1}$. When considering the solutions below we will often plot only the element $q_{1}$, or a measure dependent on $q_{1}$, since this is directly related to the amount of order along the main director, which is in the $x$ direction. The scalar order parameter in this direction is then $S=3 q_{1} / 2$. It should be noted at this point that although our calculations always find a solution for which $q_{2} \equiv 0, q_{3} \equiv 0$ and $q_{5} \equiv 0$, so that the order tensor is always of the form given in (3), we have not constrained $\mathbf{Q}$ to be so. We will however assume that variation in $\mathbf{Q}$ only occurs in the $z$ direction.

Taking the total free energy $\mathcal{F}$ (per unit area in the $x y$-plane) of the system to be the integral over the region of the sum of the thermotropic, $F_{t}$, distortion, $F_{d}$, and electrostatic, $F_{e}$, energy densities together with the surface energy, $F_{s}$, evaluated at the two substrates, gives

$$
\begin{aligned}
\mathcal{F} & =\int_{0}^{d}\left(F_{t}+F_{d}+F_{e}\right) d z+\left.F_{s}\right|_{0}+\left.F_{s}\right|_{d} \\
& =\int_{0}^{d}\left(a \operatorname{tr}\left(\mathbf{Q}^{2}\right)+\frac{2 b}{3} \operatorname{tr}\left(\mathbf{Q}^{3}\right)+\frac{c}{2} \operatorname{tr}\left(\mathbf{Q}^{2}\right)^{2}\right. \\
& +\sum_{i=1,2,3}\left[\frac{L_{1}}{2}\left(\frac{\partial Q_{i i}}{\partial z}\right)^{2}+\frac{L_{2}+L_{3}}{2}\left(\frac{\partial Q_{i 3}}{\partial z}\right)^{2}\right] \\
& \left.-\frac{1}{2} \varepsilon_{0}(\varepsilon \mathbf{E}) \cdot \mathbf{E}\right) d z+\left.W_{0}\left(\mathbf{Q}-\mathbf{Q}_{0}\right)^{2}\right|_{0}+\left.W_{0}\left(\mathbf{Q}-\mathbf{Q}_{0}\right)^{2}\right|_{d}
\end{aligned}
$$

where $d$ is the extent of the region in the $z$-direction, $\mathbf{E}$ is the electric field, $\varepsilon$ is the dielectric permittivity tensor and $\mathbf{Q}_{0}$ is the preferred value of the tensor order parameter at the substrate. The form of these energy densities has been discussed many times before [4-6] and we shall simply summarise the main features of each energy term here.

The thermotropic energy density, $F_{t}$, is a Taylor expansion of the actual energy density about the isotropic phase $\mathbf{Q}=0$. The absence of a term linear in $\mathbf{Q}$ is due to the fact that the order tensor is traceless. The coefficients $a, b$ and $c$ will be temperature dependent although it is usual to assume that $b$ and $c$ are largely independent and $a$ is linearly dependent on temperature. We take $a=\alpha\left(T-T^{*}\right)$ where $T^{*}$ is the supercooling temperature, below which the isotropic phase is unstable [4].

The distortion energy, $F_{d}$, is also an expansion of the true elastic energy, assuming that higher derivatives of $\mathbf{Q}$ and higher powers of $\mathbf{Q}$ and its derivatives are negligible compared to the leading order terms. The number of terms to leading order has been reduced using the symmetry properties of the nematic phase [5].

The electrostatic energy density, $F_{e}$, is derived from the integral of D.E, the scalar product of the displacement field and the electric field. We assume the relationship $\mathbf{D}=$ 
$\varepsilon_{0} \varepsilon \mathbf{E}$, where $\varepsilon_{0}$ is the permittivity of free space and $\varepsilon$ is the dielectric permittivity tensor of the material, which can be written (to leading order) as $\varepsilon=\bar{\varepsilon} \mathbf{I}+\Delta \varepsilon^{*} \mathbf{Q}$. Here $\bar{\varepsilon}$ is the permittivity of the isotropic phase while $\Delta \varepsilon^{*}=\Delta \varepsilon / S_{e q}$ is the rescaled dielectric anisotropy of the nematic phase, that is, the experimentally measured anisotropy $\Delta \varepsilon$ divided by the bulk nematic scalar order parameter $S_{e q}$ when the measurement was performed.

One of Maxwell's equations for a static system, $\nabla \times \mathbf{E}$, is utilised in order to express the electric field in terms of an electric potential, $\mathbf{E}=\left(0,0,-\frac{\partial U}{\partial z}\right)$, whilst another of Maxwell's equations, $\nabla . \mathbf{D}$, is used to find a solution for the electric potential. This approach is equivalent to using the Euler-Lagrange equation from the minimisation of the free energy (4) with respect to $U$.

The surface energy in (4) is the simplest form that exhibits a minimum at the preferred orientation $\mathbf{Q}_{0}$. The depth of this minimum is controlled by the anchoring strength $W_{0}$. For homogeneous alignment in the $x$ direction, the form of $\mathbf{Q}_{0}$ is taken to be

$$
\mathbf{Q}_{0}=\left(\begin{array}{ccc}
0.4 & 0 & 0 \\
0 & -0.2 & 0 \\
0 & 0 & -0.2
\end{array}\right)
$$

so that the substrate prefers an amount of order equivalent to $S=0.6$.

For the total energy $\mathcal{F}$ given by (4), the governing differential equations for the $q_{i}$ values are then the Euler-Lagrange equations, the solution of which minimises the free energy. The Euler-Lagrange equations for the five elements of $\mathbf{Q}$ are (for $i=1 \ldots 5$ )

$$
0=\frac{\partial F}{\partial q_{i}}-\frac{\partial}{\partial z}\left(\frac{\partial F}{\partial\left(\frac{\partial q_{i}}{\partial z}\right)}\right)
$$

and the equation for the electric potential is

$$
0=\frac{\partial F}{\partial U}-\frac{\partial}{\partial z}\left(\frac{\partial F}{\partial\left(\frac{\partial U}{\partial z}\right)}\right)
$$

where $F=F_{t}+F_{d}+F_{e}$ is the bulk energy density.

The boundary conditions for the $q_{i}$ equations, derived from the minimisation of the total free energy, are

$$
\pm \frac{\partial F}{\partial\left(\frac{\partial q_{i}}{\partial z}\right)}=\frac{\partial F_{s}}{\partial q_{i}}
$$

where $i=1 \ldots 5$ and the plus and minus signs correspond to the substrate at $z=0$ and $z=d$ respectively. For the electric potential we assume a potential difference of $V$ is applied across the substrates so that

$$
\begin{array}{ccc}
U=0 & \text { on } & z=0 \\
U=V & \text { on } & z=d
\end{array} .
$$


The parameters used in this model are $L_{1}=9.7 \times 10^{-12} \mathrm{~N}, L_{2}=3.5 \times 10^{-12} \mathrm{~N}$, $L_{3}=0.0 \mathrm{~N}, b=-1.6 \times 10^{6} \mathrm{Nm}^{-2}, c=3.5 \times 10^{6} \mathrm{Nm}^{-2}, \varepsilon_{0}=8.854 \times 10^{-12} \mathrm{Fm}^{-1}, \bar{\varepsilon}=8.33$ and $\Delta \varepsilon=-3$ which are typical values for a nematic liquid crystal. The parameters $a, W_{0}$ and $V$ take various values and will be specified when needed. For the thermotropic energy in (4) there are three critical values of $a$ as temperature (and therefore $a$ ) is reduced: $a=$ $b^{2} / 24 c=0.0305$ when the nematic phase first becomes locally stable; $a=b^{2} / 27 c=0.0271$ when the nematic and isotropic states have the same energy; and $a=0$, at which point the isotropic phase becomes unstable.

\section{NUMERICAL SOLUTION OF GOVERNING EQUATIONS}

To solve the Euler-Lagrange equations (6) and (7) together with the boundary conditions (8) and (9) numerically, we introduce a 'time' derivative and then relax to a steady-state solution, discretising in time via a semi-implicit Euler method. This time-dependent equation is equivalent to a balance of dissipative energy and free energy variations [7], where dissipation is derived from director motion and order changes rather than viscous flow. Second-order central finite differences are used to evaluate the spatial derivatives in the bulk of the sample and, at the boundaries, second-order left-handed and right-handed differences are used.

We consider that a steady-state solution has been reached when the difference in the scaled 2-norm of the solution from one time step to the next is below a certain tolerance set by the user depending on the accuracy required. The scaled 2-norm of a vector $\mathbf{v}$ is defined as

$$
\|\mathbf{v}\|_{2}=\sqrt{\frac{1}{N} \sum_{j=1}^{N}\left(v_{j}\right)^{2}}
$$

where $v_{j}$ is the $j$ th entry of $\mathbf{v}$ and $N$ is the length of the vector $\mathbf{v}$. For the above test, the vector used contains the values of all the the dependent variables on the computational grid.

After determining that a steady state has been reached, we then ensure that a minimum energy state has been reached by using numerical quadrature to calculate the free energy and the local curvature of the free energy surface. If the curvature is positive, we have found a minimum energy state. This final check is used to make sure that we are not either at a maximum in the energy (which would also satisfy the Euler-Lagrange equations) or on an energy plateau where the change in solution may be small while a minimum has not been reached.

To find a sequence of solutions as a particular parameter $\left(a, W_{0}\right.$ or $\left.V\right)$ varies, we use a simple form of continuation, that is, we use the solution at the previous parameter value as the initial guess for the next computation. 


\section{RESULTS}

Figure 2 shows three possible solutions of the governing equations for parameter values $a=0.0282 \times 10^{6} \mathrm{Nm}^{-2}, W_{0}=3.0 \times 10^{-5} \mathrm{Nm}^{-1}$ and $V=0$ volts. We can now classify solutions at other parameter values with reference to these three solutions: the isotropic solution (I) where the bulk of the cell is in the isotropic state with the ordering substrates inducing partial wetting of the nematic state (also termed a paranematic state); the nematic solution $(\mathrm{N})$ where the bulk of the cell contains a nematic phase, with the order parameter equal to the equilibrium value determined by the thermotropic potential except close to the substrates where enhanced ordering occurs; and the wetting solution (W) in which there are distinct layers of the nematic phase at and close to the substrates with the isotropic state in the bulk of the sample. The wetting solution is the result of an energy balance between the substrate anchoring, which prefers the boundaries to be in a nematic state, and the thermotropic energy which, at this temperature, prefers the system to be in an isotropic state.

Figure 3 shows the regions of existence of these solutions as temperature varies, using the scaled 2-norm of the vector of $q_{1}$ values on the computational grid as a solution measure, for a number of anchoring strengths. Close to critical points, where solutions lose stability, the parameter continuation step was decreased to improve accuracy. We have also marked certain important points on each graph: $N_{U}$ is the upper bound at which a nematic solution exists; $I_{L}$ is the lowest temperature at which an isotropic solution exists; $W_{U}$ and $W_{L}$ are the upper and lower bounds of the wetting solution, respectively. Note that, from the solutions in Figure 2, in the nematic state we expect the scaled 2-norm of $q_{1}$ to be around 0.1 and similarly, we expect the scaled 2-norm of $q_{1}$ to be close to zero for the isotropic state.

From Figure 3(a) and (b) we see that, for low anchoring strengths, the range of $a$ values for which the wetting solution exists $\left[W_{L}, W_{U}\right]$ is contained within the range $\left[I_{L}, N_{U}\right]$ (note that in Figure 3(a) $W_{U}$ and $W_{L}$ almost coincide). Therefore, when cooling from the isotropic state or heating from the nematic state, the wetting will never be accessed. As the anchoring strength is increased (Figure $3(\mathrm{c})$ ) the lower bound of wetting solution, $W_{L}$, and the lower bound of the isotropic solution, $I_{L}$, cross. In this case the wetting layer solution will be accessed, through a first order transition, by decreasing the parameter $a$ (in other words temperature) past $I_{L}$. By further decreasing temperature, the wetting solution will be transformed, through another first order transition, to the nematic solution. Increasing temperature will cause the nematic state to transform directly to the isotropic state. Finally, when the anchoring strength is increased further, the isotropic to wetting transition becomes continuous (Figures $3(\mathrm{~d})$ and (e)).

By plotting values of $N_{U}, I_{L}, W_{U}$ and $W_{L}$ over a large range of anchoring strengths, we can encapsulate the information in Figure 3 in a single phase diagram (see Figure 4(a)). This shows all the behaviour described above: although, for low anchoring strengths, the wetting layer is confined to a temperature region between $I_{L}$ and $N_{U}$, as anchoring is 
increased the $I_{L}$ curve crosses the $W_{L}$ curve so that the wetting solution is accessible; at higher anchoring strengths the $I_{L}$ and $W_{U}$ curves meet so that the isotropic to wetting layer transition becomes continuous. For $W_{0}=0$ we expect that no wetting solution exist.

Figure 4(b) shows the equivalent phase diagram when a voltage of 2 volts is applied to the cell. An applied electric field in the $z$ direction, when $\Delta \epsilon<0$, should increase the tendency for molecules to align in a direction perpendicular to $z$. For the present situation, because the boundaries prefer alignment in the $x$ direction, the electric field will induce order and alignment in the $x$ direction. This can be seen when comparing Figures 4(a) and (b). In the latter, the $N_{U}$ and $W_{L}$ curves have shifted upwards, indicating that the nematic and wetting layer states have been stabilised for higher temperatures. The point at which the $I_{L}$ curve meets the $W_{L}$ curve occurs at a higher temperature and lower value of $W_{0}$ implying that, even though the wetting state is stabilised for higher temperatures, the influence of both field alignment and anchoring alignment will induce the nematic state at a lower anchoring strength. This last point can be seen clearly in Figure 5, which indicates the extent of the wetting state region for voltages ranging from 0 volts to 12 volts. The range of anchoring strengths at which wetting solutions exist decreases as the voltage is increased until the wetting state does not exist at 12 volts. This decrease in the range of anchoring strengths over which the wetting state exists is due to the combined aligning effects of the applied field and the anchoring substrates inducing enough order to transform the wetting state into the nematic state. The circles marked in Figure 5 indicate the points at which the $I_{L}$ and $W_{L}$ curves cross in Figure 4. As discussed above, the wetting state can only be accessed (by reducing temperature whilst in the isotropic state) when the $I_{L}$ curve is above the $W_{L}$ curve, or equivalently, to the right of the circle in Figure 5.

\section{SUMMARY}

We have investigated the existence of wetting layers in a nematic confined between two aligning substrates as the anchoring strength, temperature and applied voltage are varied. The range of temperatures over which wetting solutions exist was found to initially increase as anchoring strength increased but then decrease until the isotropic to wetting layer transition transformed from a first order to continuous transition. With the introduction of an electric field, which induces additional order in the system, we found that the nematic state can occur at higher temperatures and with a sufficiently high voltage the wetting layer regime is destroyed.

\section{REFERENCES}

[1] Sluckin, T.J., Poniewierski A., in Fluid Interfacial Phenomena., John Wiley \& Sons Ltd, (1986).

[2] Lelidis I. Liquid Crystals, 25, 531, (1998).

[3] Teixeira P.I.C., Sluckin T.J., Sullivan D.E. Liquid Crystals, 14, 1243, (1993).

[4] E.G. Virga: Variational Theories for Liquid Crystals, Chapman \& Hall, London, (1994). 
[5] De Gennes P.G., Prost J. The Physics of Liquid Crystals: 2 ed., Clarendon Press, Oxford, (1993).

[6] Schophol N., Sluckin T.J. Physical Review Letters, 59, 2582, (1987).

[7] Sonnet A.M., Maffettone P.L., Virga E.G. Journal of Non-Newtonian Fluid Mechanics, 119, 51, (2004). 

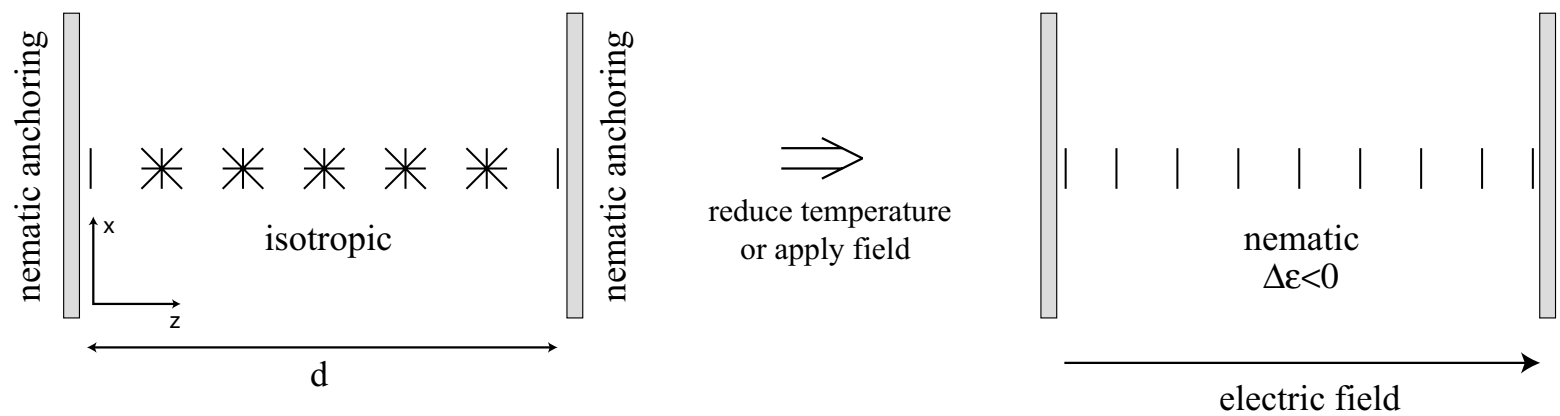

Figure 1: Schematic of the cell configuration: an isotropic material is sandwiched between two aligning substrates, a distance $d$ apart. The application of an electric field and/or a reduction in temperature will induce a phase change. 


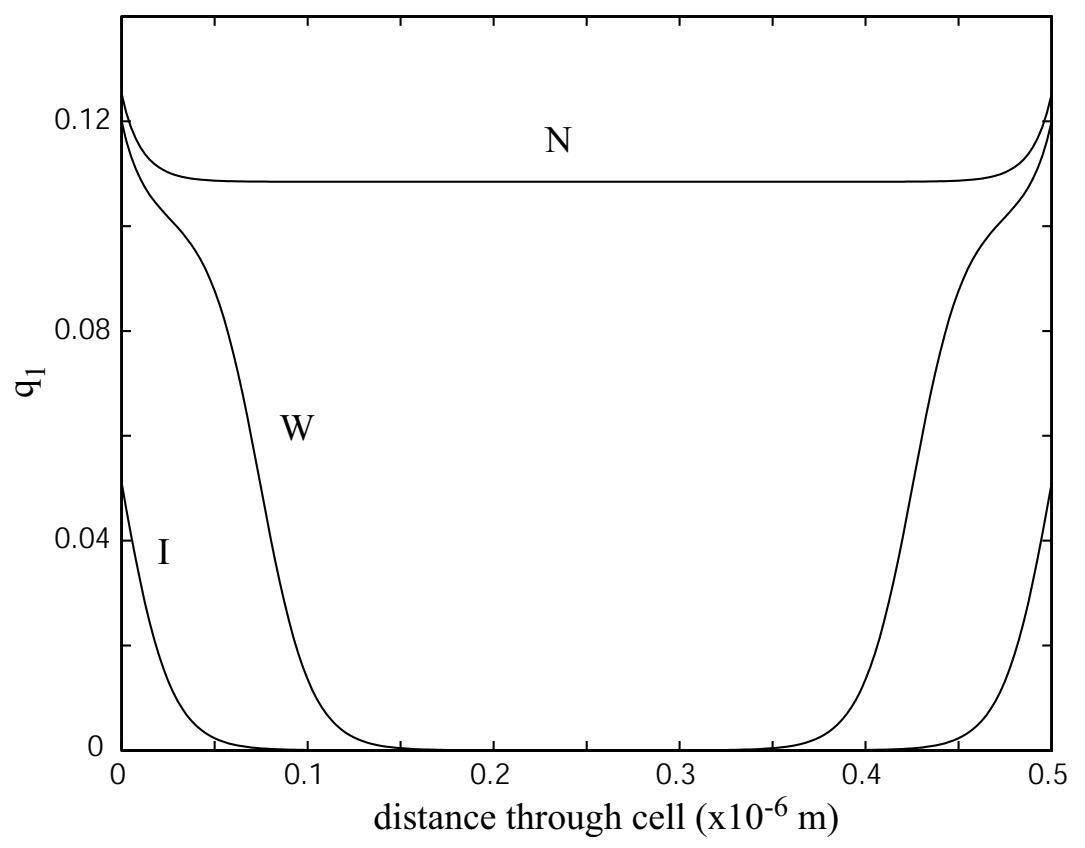

Figure 2: Solutions for $q_{1}(z)$ : the isotropic solution (I), nematic solution $(\mathrm{N})$ and wetting solution (W) for $a=0.0282 \times 10^{6} \mathrm{Nm}^{-2}, W_{0}=3.0 \times 10^{-5} \mathrm{Nm}^{-1}$ and $V=0$ volts. 
(a)
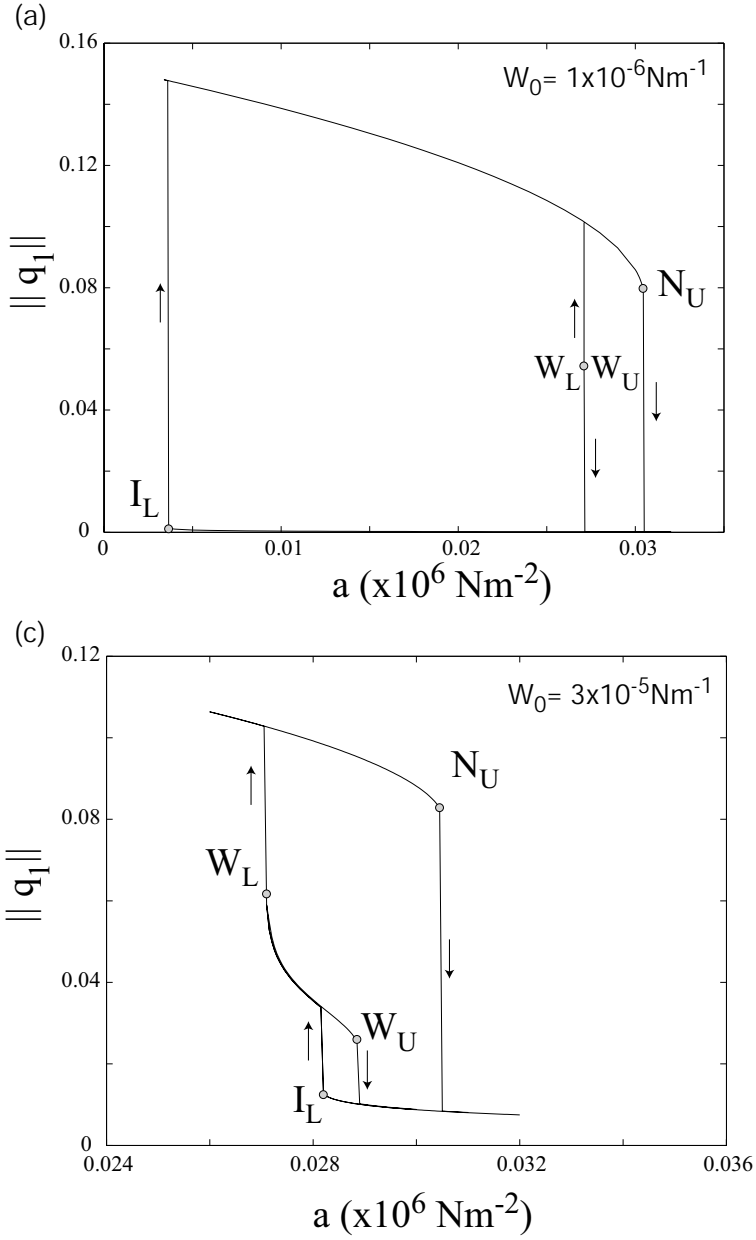

(b)
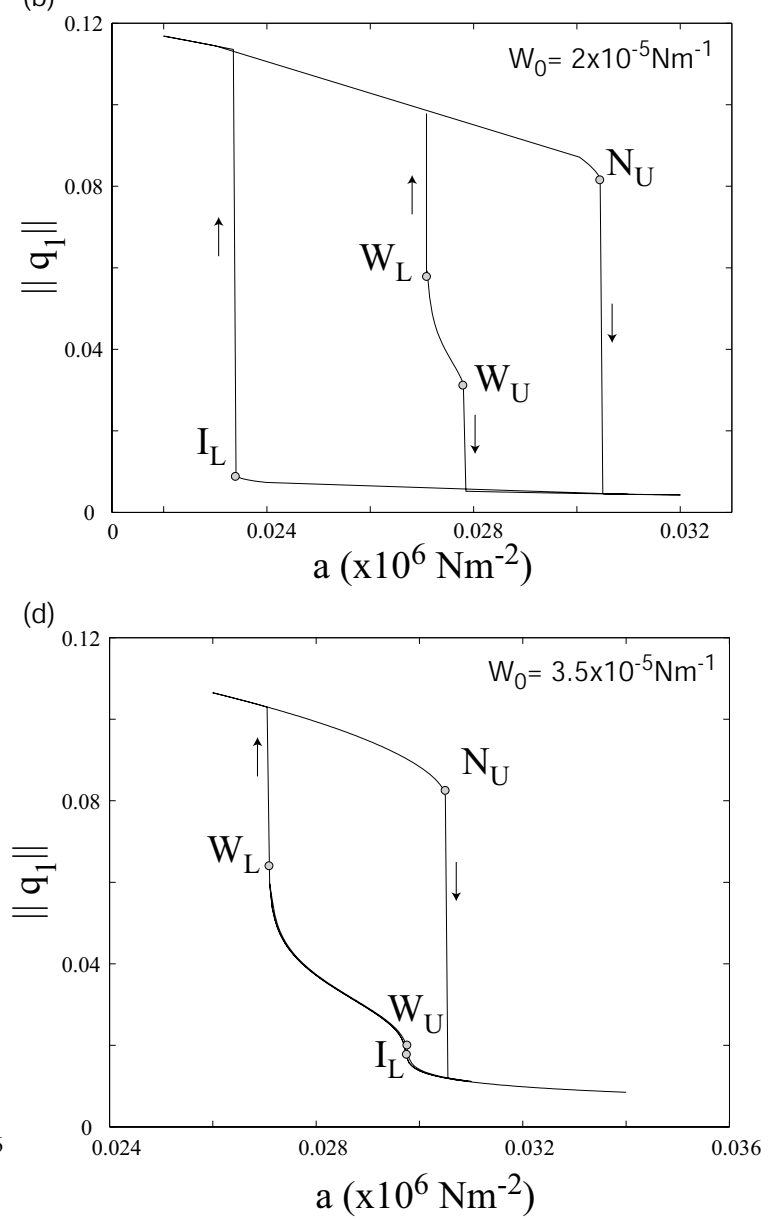

(e)

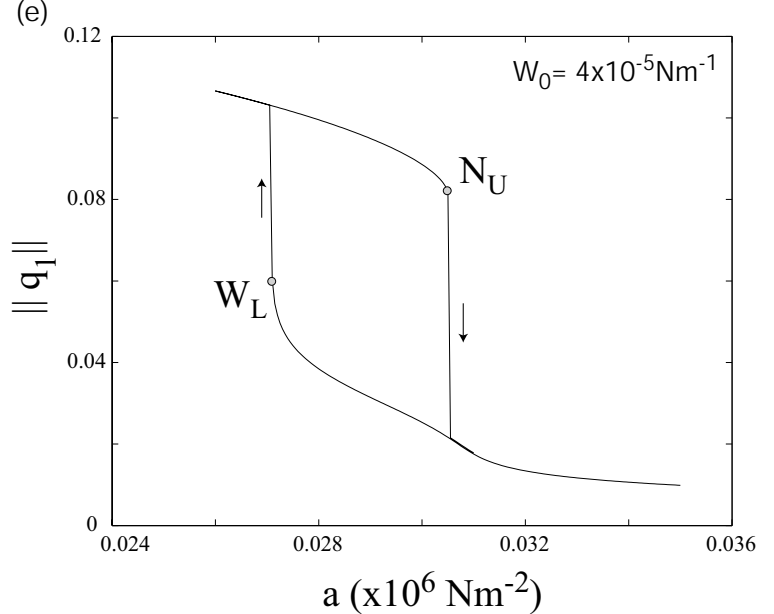

Figure 3: Plots of the scaled 2-norm of $q_{1}$ against $a$ (temperature) for various surface anchoring strengths and for $V=0$ volts 

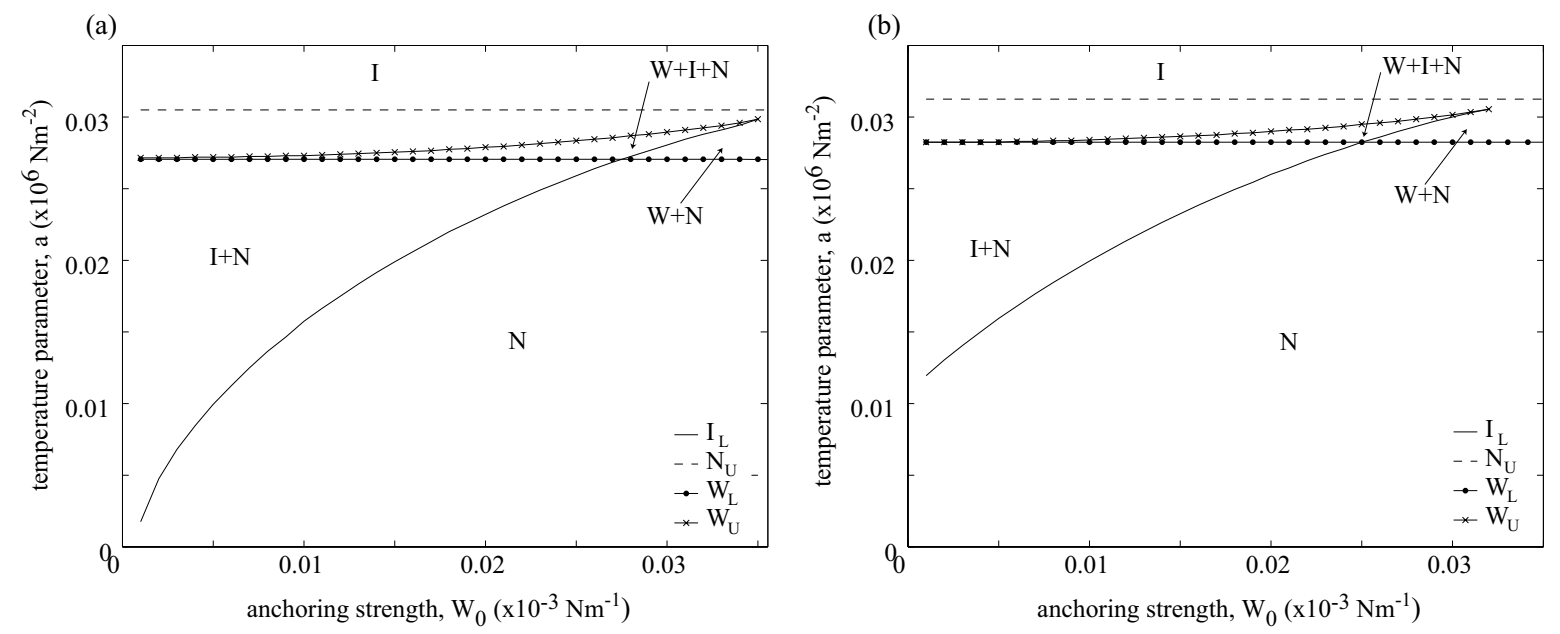

Figure 4: Phase diagrams as temperature, $a$, and anchoring strength, $W_{0}$, are varied: (a) $V=0$ volts, (b) $V=2$ volts. The labels $I, W$ and $N$ indicate the regions in which the isotropic, wetting layer and nematic states are stable. 


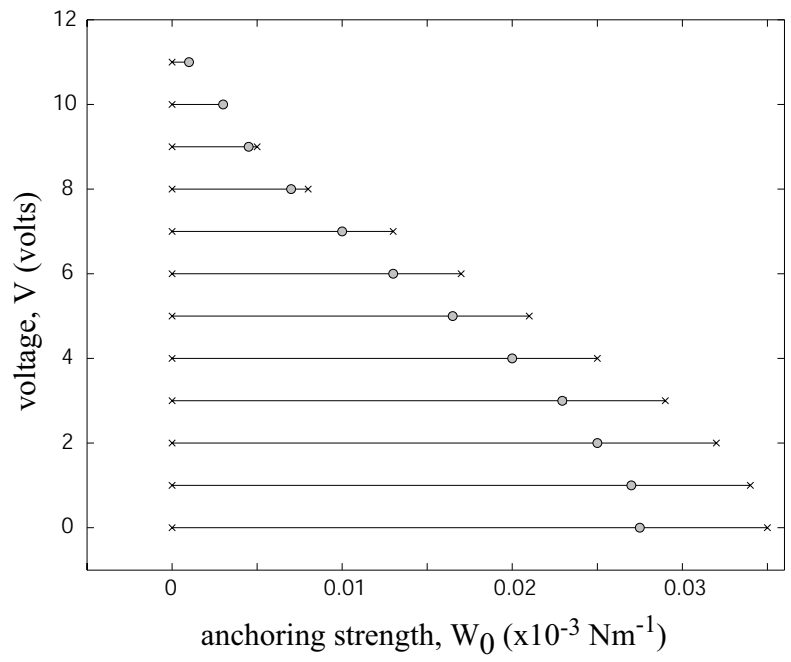

Figure 5: Extent of the wetting state region as a function of anchoring strength and voltage. The circle indicates the anchoring strength at which $I_{L}=W_{U}$. 